\title{
La accesibilidad un derecho de la colectividad, en áreas de interés patrimonial, como reconocimiento de una parte de la inclusión social, ante las barreras del espacio. Caso de estudio: Área Central de Mérida, Venezuela
}

LEYDA COROMOTO RONDÓN VELÁZQUEZ

$>$ Arquitecta. Red Internacional de Promotores ODS, Venezuela

leydarondon@gmail.com

ORCID 0000-0003-1096-5423

Universidad de Valparaíso

Facultad de Arquitectura

Revista Márgenes

Espacio Arte Sociedad

La accesibilidad un derecho de la colectividad, en áreas de interés patrimonial, como reconocimiento de una parte de la inclusión social, ante las barreras del espacio. Caso de estudio: Área Central de Mérida, Venezuela

Diciembre 2021 Vol $14 N^{\circ} 21$

Páginas 101 a 112

ISSN electrónico 0719-4436

Recepción mayo 2021

Aceptación julio 2021

DOI 10.22370/margenes.

2021.14.21.3102
RESUMEN

En la actualidad, se ha generado una brecha, entre las legislaciones existentes en América Latina y la implementación de estas normativas, especialmente al grave problema de accesibilidad universal en áreas con intereses patrimoniales, que aleja cada vez más a las personas con diversas limitaciones para su movilidad.

La búsqueda consiste en políticas públicas que logren cubrir ámbitos acompañados de la participación, la igualdad, el empleo, la educación y la formación, permitiendo que las personas puedan estudiar, recrearse, desplazarse o disfrutar de un servicio de salud. En este trabajo, se plantea un mecanismo de políticas públicas inclusivas, seguras, resilientes y sostenibles, orientadas a formular y desarrollar planes estratégicos de accesibilidad, estimulando su materialización en las diferentes escalas territoriales. Logrando realizar un análisis descriptivo de los términos de discapacidad como derecho y no como beneficio que otorga el Estado, desarrollado en el área de estudio, casco central de la ciudad de Mérida, para finalmente, establecer como conclusión, que se requiere el desarrollo de una planificación estratégica junto a una coordinación de sus políticas de rehabilitación de sus espacios públicos y una regularización de las edificaciones y del mobiliario urbano existente, en especial, el patrimonial, en función de la igualdad de condiciones. PALABRAS CLAVE

políticas públicas, inclusión social, planificación estratégica, accesibilidad universal, barreras del espacio

Accessibility is a right of the community, in areas of patrimonial interest, as recognition of a part of social inclusion, before the barriers of space. Case study: Central Área of Mérida, Venezuela ABSTRACT

Currently, a gap has been created between the existing legislations in Latin America and the implementation of these regulations, especially the serious problem of universal accessibility in areas with patrimonial interests, which increasingly alienated people with various limitations for their mobility.

The search consists of public policies that manage to cover areas accompanied by participation, equality, employment, education and training, allowing people to study, recreate, move or enjoy a health service. In this paper, a mechanism of inclusive, safe, resilient and sustainable public policies is proposed, aimed at formulating and developing strategic accessibility plans, stimulating their materialization in the different territorial scales. Managing to carry out a descriptive analysis of the terms of disability as a right and not as a benefit granted by the State, developed 
in the study area, central area of the city of Mérida, to finally establish as a conclusion, that the development of a strategic planning is required together with a coordination of its policies of rehabilitation of its public spaces and a regularization of the buildings and the existing urban furniture, in particular, the patrimonial, depending on the equality of conditions.

\section{KEYWORDS}

public policies, social inclusion, strategic planning, universal accessibility, space barriers

\section{INTRODUCCIÓN}

Toda persona demanda de la sociedad el derecho a una vida en igualdad de condiciones, sin embargo, durante su desarrollo y crecimiento se van encontrando con una serie de barreras o limitaciones que disminuyen sus condiciones, descubriendo espacios o servicios de salud, educativos y recreativos, que impiden lograr tener una vida decorosa y de buena calidad, donde se les permita participar y contribuir del desarrollo socioeconómico y cultural de su comunidad, es decir, donde exista una mejor inclusión social, disminuyendo problemas tanto en el traslado como dentro de las infraestructura.

Llevar a consolidar la plena integración de las personas con discapacidad como una cuestión de derecho y no como un privilegio que otorga el Estado, es más, un cambio cultural que induce a obtener de la sociedad una visión diferente a la actual.

Las tendencias actuales de discapacidad en América Latina, son estadísticas desactualizadas y poco precisas, sin embargo, en un estudio coordinado por la Organización Panamericana de la Salud (1998), sobre salud, bienestar y envejecimiento en siete ciudades: Buenos Aires (Argentina), Bridgetown (Barbados), Sao Paulo (Brasil), Santiago de Chile (Chile), La Habana (Cuba), Ciudad de México (México) y Montevideo (Uruguay), encontró que en América Latina existen aproximadamente 85 millones de personas con discapacidad, donde más de un cuarto de la población total de la región se encuentre afectada directa o indirectamente por la discapacidad de familiares, amistades o miembros de la comunidad,... caracterizados por extrema pobreza, tasa elevada de desempleo, acceso limitado a los servicios públicos de educación, atención médica, vivienda, transporte, legales y otros, derivando problemas en cuanto a la deserción en la educación y el alto grado de desempleo para personas discapacitadas, unido a la falta de programas de prevención y detección temprana. En consecuencia, debemos destacar que alrededor del 50\% de la población con discapacidad en la región tiene edad para trabajar (entre 2.250.000 y 2.956.000 personas), lo que nos obliga a encontrar respuestas eficientes, que les otorguen la accesibilidad a estas personas (Vásquez, p. 10, 11 y 12).

En este sentido, para que una persona pueda incorporarse al mercado laboral y a las actividades cotidianas, deberán de superarse algunos problemas que limitan su pleno desarrollo, definidas como barreras del espacio construido, considerando que la Organización Mundial de la Salud ONU (1999:38) establece que:

El desarrollo de las condiciones necesarias para que exista una igualdad de oportunidades puede reducir las barreras que dificultan la integración social y la incorporación al desarrollo de las personas con discapacidad, permitiendo que el sistema general de la sociedad, es decir, el medio físico y cultural, la vivienda, el transporte, los servicios sociales y sanitarios, las oportunidades de educación y trabajo, así como la vida cultural y social, se vuelva accesible para todos (Bojórquez, 2006:45).

Partiendo de que la mayoría de los países en Latinoamérica, tienen sus áreas centrales, desarrolladas bajo el contexto de valor tradicional y que poseen legislaciones que involucran la accesibilidad como política pública, se implanta como hipótesis: que debido a una falta de coordinación entre los diferentes servicios e instituciones del Estado, no se ha podido desarrollar el espíritu de la Ley sobre estos espacios originales, antiguos y tradicionales, que permita ver materializada su intención, imposibilitando dar respuestas eficientes en el uso de los recursos existentes, para el logro de una accesibilidad universal, referidos a un protocolo de acción para emprender dicha labor, donde se logre la disminución de barreras, no solo arquitectónicas o de acceso al espacio físico, sino también, el uso y disfrute de espacios que constituyen un conjunto patrimonial diverso y complementario; derivando al mismo tiempo, una segunda hipótesis: que considera, que la relación entre una persona con discapacidad y el territorio, es decir, la movilidad, es el efecto primordial para estimular el resto de la accesibilidad en un área de salud, educacional, cultural o laboral, interacción que permitiría aumentar la autoestima de la persona con limitaciones y estimular su incorporación al resto de la colectividad.

Concretándose como objetivo general del trabajo, Identificar un mecanismo eficiente en donde la colectividad, desarrolle una accesibilidad universal, que lleven a las áreas centrales y con valor tradicional de las ciudades latinoamericanas, a un nuevo equilibrio, basado en la inclusión social, a través de la interacción entre el ser humano y el territorio, ante la existencia de barreras en el espacio construido, donde el área de estudio, corresponde al área central de la ciudad de Mérida del Estado Mérida, Venezuela, ciudad tradicional y turística. 


\section{ESTADO DEL ARTE}

Para comprender el alcance de cada término utilizado dentro de este trabajo se procede a aclarar la definición de inclusión social:

la CEPAL (2014) define a la inclusión social como el proceso por el cual se alcanza la igualdad, y como un proceso para cerrar las brechas en cuanto a la productividad, a las capacidades (educación) y el empleo, la segmentación laboral, y la informalidad, que resultan ser las principales causas de la inequidad (Muñoz \& Barrantes, 2016:17).

En este sentido la inclusión social se distingue con la accesibilidad por lo generalizado que es este término, para ello la accesibilidad según la Comisión Europea CEAPAT (1996) es:

Una característica básica del entorno construido. Es la condición que posibilita el llegar, entrar, salir y utilizar las casas, las tiendas, los teatros, los parques y los lugares de trabajo. La accesibilidad permite a las personas participar en las actividades sociales y económicas para las que se ha concebido el entorno construido (Linares et al., 2018:117).

Tomando en consideración que dicha accesibilidad se ve interrumpida con las llamadas Barreras del espacio, produciendo ghetos de exclusión Olivera, (2006) afirma que:

La discapacidad y las barreras creadas por el espacio natural y por el espacio construido son exclusógenos especialmente graves. Producen exclusión económica, educativa, de las redes sociales, de la participación ciudadana, del ocio, etc.., en parte por las propias barreras psicológicas de los afectados, pero sobre todo por la exclusión territorial que causa la sociedad al vetar, muchas veces inconscientemente, el uso del espacio a algunos habitantes, que quedan desterrados de los espacios de la vida cotidiana, recluidos en su propia vivienda o en instituciones (Linares, Hernández \& Rojas, 2018:118).

\section{El derecho de las personas con discapacidad, por encima de un simple beneficio que otorga el estado}

Entendiendo la discapacidad según la CIF (Clasificación Internacional del Funcionamiento de la salud) (2011), como el resultado de la interacción entre personas con diferentes niveles de funcionamiento y un entorno social que no toma en cuenta esas diferencias, se puede afirmar que la discapacidad se produce de acuerdo a la brecha o la interacción que exista entre de la persona y ese entorno social. El término no depende de la causa de la discapacidad, sino que nos hace pensar en los componentes del funcionamiento humano (Stang, 2011:11).

Para ello tenemos que conocer de las categorías de la clasificación de discapacidad, que nos presenta la CIF por medio de la Organización Mundial de la Salud (Clasificación Internacional del Funcionamiento de la salud) (2001), en donde se refieren a los siguientes tipos de discapacidades:

1. Discapacidad física o motora: que produce debilidad o limitación en los movimientos físicos.

2. Discapacidad sensorial visual: discapacidad en la vista.

3. Discapacidad sensorial auditiva: discapacidad para escuchar.

4. Discapacidad cognitiva: la que refiere a capacidades cognitivas y de aprendizaje, incluyendo enfermedades neurológicas, de conducta y de salud mental (Banco Interamericano de Desarrollo, 2019:9).

Para garantizar el acceso pleno a las personas con discapacidad, existen las políticas públicas que permiten orientar la gestión de un gobierno, con la finalidad de alcanzar su objetivo, siendo la accesibilidad una forma de inclusión a la sociedad, correspondiendo a la vez un tema cultural, que abarca las distintas formas y expresiones de una sociedad determinada.

En algunos países disponen de una institucionalidad a cargo de las personas con discapacidad, sin embargo, su forma de operar sus acciones o estrategias continúan representando un problema que demanda un análisis y acciones estructurales, que abarcaría intereses de los poderes económicos y políticos comprometidos con el nuevo modo de estructuración social y los factores sociales de la ignorancia, el abandono, la superstición y el miedo, que a lo largo de la historia han aislado y han retrasado su desarrollo (Naciones Unidas, 1994), en Latinoamérica se ha podido observar la existencia de legislaciones y estrategias que solo quedan como propuestas, sin ofrecer resultados y mucho menos estar incluidas en las estructuras sociales en búsqueda de una reducción de la discriminación, la segregación y la exclusión (Stang, 2011:63).

La desigualdad que reciben estas personas, por parte de organismos internacionales y los gobiernos se profundiza desde comienzos de la década de 1990, pasando desde un enfoque que consideraba a las personas con discapacidad como víctimas, objetos de caridad y beneficiarios de programas, hacia una conceptualización que las concibe como sujetos de derechos, participantes y actores, reconociendo su contribución a la sociedad y reclamando su integración (Savedoff, 2006; Stang, 2011:7).

La manera con la que se observa y se aborda esta problemática de la desigualdad y la vulnerabilidad que poseen las personas con discapacidad, va a marcar la diferencia entre la forma de gestionar de cada gobierno. A nivel Internacional se ha logrado un cambio en la Clasificación Internacional del Funcionamiento, de la Discapacidad y la Salud (CIF), de la Organización Mundial de la Salud (OMS), vigente desde 2001, donde la CIF define a la discapacidad como un continuo multidimensional de capacidades funcionales, más que como una lista de condiciones específicas a una categoría, a una minoría o a un grupo particular (Savedoff, 2006) midiendo la discapacidad en función del grado en que esa capacidad es limitada (Stang, 2011:8)

Según informe del Secretario General de la Naciones Unidas sobre el grado de cumplimiento de los Objetivos de Desarrollo del Milenio (ODM) (2009), encontramos los siguientes datos sobre esta problemática:

426 millones de personas con discapacidad viven por debajo del umbral de la pobreza en los países en desarrollo, representando entre el $15 \%$ y el $20 \%$ de la población pobre más marginada de estos países. Evidenciando que su participación en la fuerza de trabajo es muy inferior a la de las personas sin discapacidad. La Organización de la Naciones Unidas para la Educación, la Ciencia y la Cultura (UNESCO) estima que más del 90\% de los niños con discapacidad de los países en desarrollo no asiste a la escuela (Stang, 2011:8)

En definitiva, este informe del secretario General de las Naciones Unidas sobre el nivel de cumplimiento de los Objetivos de Desarrollo del Milenio, describe un escenario de escasez de datos y am- 
plias diferencias entre las definiciones, las normas y las metodologías utilizadas para determinar las condiciones de las personas con discapacidad, situación que obstaculiza la eficaz formulación de políticas y programas inclusivos, pero que a la vez nos incita a formular una mejor situación (Stang, 2011:8).

\section{EXPERIENCIAS DEL MANEJO DE LA ACCESIBILIDAD UNIVERSAL EN EUROPA Y AMÉRICA LATINA}

\section{Accesibilidad en Europa}

Europa inicia estudios sobre accesibilidad a partir de los años 70 respaldando la definición de accesibilidad de la CEAPAT (1996) anteriormente visualizada. Tesis con la cual, se inicia en Estados miembros de la Unión Europea un proyecto denominado Estrategia Europea sobre Discapacidad 2010-2020: el cual se denominó un compromiso renovado para una Europa sin barreras (Comisión Europea, 2010) teniendo como áreas de actuación: la accesibilidad, la participación, la igualdad, el empleo, la educación y la formación, la protección social, sanidad y acción exterior, apoyando aspectos de sensibilización, apoyo financiero; y generación de estadísticas, recopilación y seguimiento de datos. De esta manera, promocionaron la implementación de mejores prácticas en las ciudades de la Unión, el cual fue estimulado a través del Premio a la Ciudad Accesible. Para el 2016, florecen dos nuevas categorías de premiaciones: Smart City, como reconocimiento a la tecnología para mejorar las condiciones de vida de las personas con discapacidad y adultos mayores y el Acceso al Trabajo, que facilitan el acceso al mercado laboral (Linares et al., 2018:6).

De igual manera, en la Unión Europea (2015), se produjo estímulos a la participación del sector privado, la aplicación de estándares de accesibilidad al entorno construido que va más allá de lo legal y que garantizan una vida independiente (Linares et al., 2018:8).

Ciudades ganadoras de España, Austria, Alemania, Suecia e Italia, se destacan por la intervención en:

- El espacio público, haciendo especial énfasis en la accesibilidad a los centros históricos de las ciudades antiguas.

- Cambios en el transporte público, libre de barreras mediante el uso de rampas extensibles.

- La tecnología de bajada del piso y sistemas de información diseñados para personas con discapacidad.

- Bases de datos gratuitas que responden a marcos políticos y presupuestarios de la ciudad.

- Recursos de apoyo para la planificación de los viajes a través de la ciudad.

- Superar los estándares de accesibilidad en el entorno construido e involucrar al sector privado.

- Planear a la ciudad a partir del concepto "ciudad para todos" destinando presupuesto para la realización de obras públicas relacionadas con la accesibilidad al espacio público, a los edificios, a la integración social, seguridad y calidad de vida de los ciudadanos (Linares et al., 2018:8).

Por último, el proyecto que emprendió la Unión Europea, tuvo su éxito al trabajo en conjunto, visto desde un concepto global, que garantizó la dignidad y la autonomía de todos los ciudadanos sin distinción, con la eliminación de sus barreras, que fueron exigidos debido al envejecimiento de la población europea que le permitirían su integración a la vida diaria.

\section{Inclusión social en América Latina}

A partir de la Convención Internacional de Derechos de las personas con discapacidad (2006), se adelantan estrategias de los sistemas de transporte e infraestructura, acciones que promueven los derechos de las personas con discapacidad en países como México, Argentina, Chile, Brasil y Colombia. (Linares et al., 2018:09).

En primer lugar, tenemos el trabajo de Hernández (2012) en México, quien valora los planteamientos de la accesibilidad a nivel teórico y conceptual, aportando un instrumento de medición y devela los obstáculos presentes en los recorridos cotidianos de las personas con discapacidad, así como las estrategias que emplean estos sujetos para apropiarse física y simbólicamente del espacio. De esta caracterización concluye que la ciudad de México, es poco accesible pues presenta grandes barreras arquitectónicas, no obstante, cuenta con una red de apoyo para la atención y rehabilitación de las personas con discapacidad. (Linares et al., 2018:10).

En este trabajo se deduce que los diagnósticos son fundamentales y se desarrollan frente al acceso a diferentes derechos, como el erguido a la educación, salud, empleo, movilidad y recreación. Construye conocimiento para la inclusión de personas con discapacidad en el turismo, transformando el lugar en el que habitan diferentes seres humanos, con y sin discapacidad, considerando que los procesos de re-ordenamiento son los instrumentos que deben procurar reducir la exclusión social históricamente naturalizada en los territorios.

\section{En Argentina}

Podemos encontrar el estudio denominado: Re-Ordenando el Territorio para el Turismo Accesible. El caso de San Martín de los Andes, Provincia de Neuquén, Patagonia Argentina, elaborado por Brinckmann y Reyes (2008). En este estudio se logró destacar la distancia que existen entre "territorialidad y espacialidad".

El colectivo social que es excluido de la sociedad es diverso y se basa en posibles cuestiones de índole sexual, económica, de raza, ideológica, religiosa, política y vinculada en lo que se refiere a lo normal y lo que se aleja de tal concepto (Linares et al., 2018:11).

En él se afirma que la sociedad inclusiva es un proceso por el que la colectividad se adapta para incluir a los discapacitados en sus sistemas sociales, y, simultáneamente, éstos se preparan para asumir sus papeles en la sociedad. La inclusión social para Brinckmann y Reyes (2008), constituye, entonces, un proceso bilateral en el que las personas aún excluidas, y la sociedad buscan resolver los problemas del individuo y del conjunto, encontrar las soluciones nivelando las oportunidades para todos (Linares et al., 2018:10).

\section{Curitiba, Brasil}

Corresponde a una de las ciudades de Latinoamérica con mayor calidad de vida, partiendo de que, en Curitiba, más de 350.000 residentes tienen algún tipo de discapacidad (Mayors Challenge, 2016), en esta ciudad realizaron una propuesta de inclusión, que se centra en el mejoramiento de los sistemas de movilidad para los ciudadanos con necesidades especiales. (Linares et al., 2018:12).

Curitiba se convierte en una ciudad incluyente materializando el principio de diseño universal al lograr consolidar para el año 2013 la accesibilidad en el 100\% de la flota de transporte, tanto de las instalaciones como de sus vehículos, están adaptados a las necesidades de toda la 
población. De igual manera, logra que la infraestructura asociada al sistema de transporte garantizará la seguridad en la movilización de las personas con necesidades especiales" (Martínez, 2012) reconociendo, que "el diseño universal les permite como política pública a los ciudadanos de Curitiba hacer efectivo su derecho a la ciudad (Linares et al., 2018:13).

\section{Bogotá, Colombia}

En el transporte Transmilenio que existe, se aplicó una metodología propuesta por la CIF de la Organización Mundial de la salud (2001), que consiste en la caracterización de perfiles de usuarios,

con la consideración de condiciones circunstanciales o temporales que podrían implicar dificultades en la movilidad cotidiana en ciertos grupos de usuarios (por ejemplo: personas con lesiones temporales que se asisten con muletas, bastones o mujeres embarazadas)". "El instrumento fue creado utilizando la herramienta Mapa de Viaje del cliente (MVC), la cual consiste en crear una visualización del proceso que sigue una persona para el logro de un propósito. Permitiendo comprender y abordar las necesidades del cliente además de identificar los puntos débiles y posibilidades de mejorar existentes dentro del proceso.

La misma consiste en los siguientes pasos:

- Planificación de la toma de información en la cual se hace la identificación de voluntarios, recopilación de información y capacitación de los entrevistadores.

- Toma de datos o trabajo de campo (en el cual se identifican las etapas de viaje).

- Procesamiento de información.

- Generación del Mapa de Viaje del Cliente MVC (BID, 2019:11).

\section{En Santiago de Chile}

Existen algunas políticas de inclusión social, como la Ley 20.422 del Ministerio de planificación (2010) que establece como objetivos: asegurar el derecho de igualdad de oportunidades de las personas con discapacidad, con el fin de obtener su plena inclusión social, asegurando el disfrute de sus derechos y eliminando cualquier forma de discriminación fundada en la discapacidad. Normas sobre la igualdad de oportunidades e inclusión social de personas con discapacidad, basándose en la accesibilidad universal, que pretende igualar los derechos de las personas discapacitadas; junto a este instrumento se encuentran la libertad de expresión, el despojo de la violencia de género. El servicio nacional de discapacidad SENADIS, quien elaboró una guía para la creación de material accesible ya sean textos, gráficos, imágenes y documentos (Castro et al., 2015:3).

Con la existencia de esta ley se ha podido establecer una comparación entre las personas estimuladas para la realización de cualquier trabajo y las que aún no se han visto favorecidas por ella, reflejando lo importante que es establecer condiciones de inclusión social en cualquier legislación (Figuras 1 y 2).

\section{LAS POLITICACAS PÚBLICAS, EL PROCESO DE URBANIZACIÓN Y LA ACCESIBILIDAD}

Dentro de la definición de ciudad se considera que,

es la integración espacial de la actividad económica y social. Las empresas se ubican cerca de otras empresas y
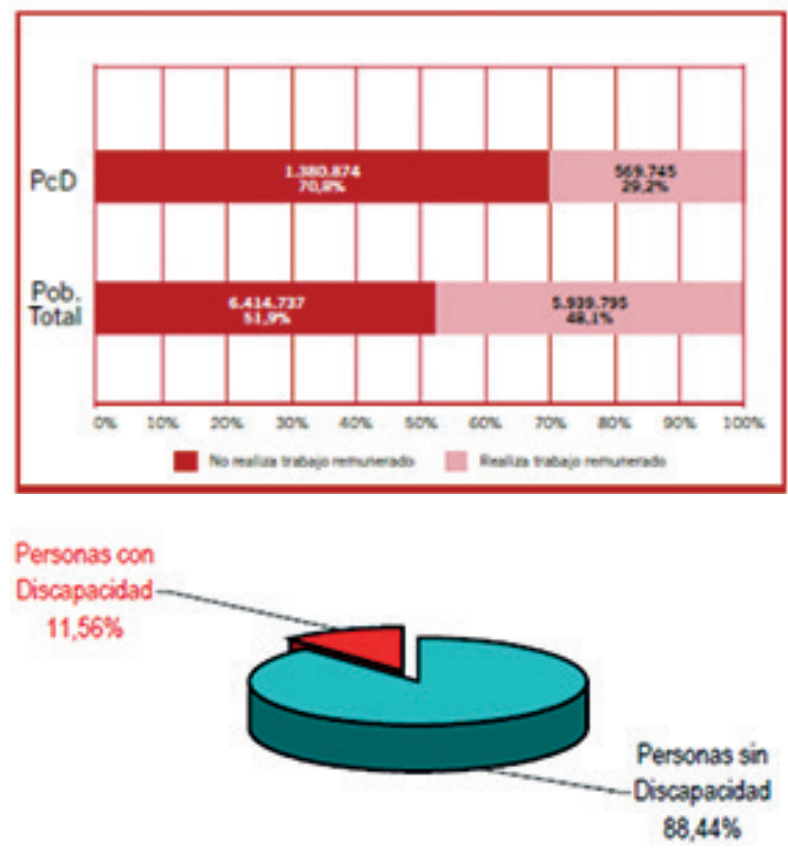

$>$ Figura 1. Discapacidad según el trabajo remunerado de las personas con y sin discapacidad Chile, 2004. Nota: En este gráfico se puede observar la distribución porcentual de las personas remuneradas con discapacidad en contraposición al resto de la población en Chile durante censo del año 2004. En donde solo el $29,2 \%$ de las personas con discapacidad realizan trabajo remunerado y un 70,8 \% no lo realiza, en comparación con el resto de la población. Tomado del Censo 2004 en Castro et al., 2015.

$>$ Figura 2. Personas con discapacidad y sin discapacidad en Chile para el 2004. Nota: Demuestra el porcentaje de población con y sin discapacidad para Chile en el 2004. Tomado del Censo 2004 en Castro et al., 2015. 
de hogares para tener un mejor acceso a insumos de producción, mano de obra y consumidores. Los hogares se ubican cerca de otros hogares y de empresas para intercambiar ideas y conocimiento $y$ tener mejores posibilidades de empleo (CAF, 2017:28).

Partiendo de que el proceso de urbanización que se lleva en una ciudad, constituye el motor de crecimiento de la economía y la productividad, es entonces, la accesibilidad el mecanismo que constituye un aspecto fundamental, para soportar las diversas escalas de concentración poblacional y de empleo, resultando también como indicador de bienestar de la población, en donde las políticas públicas podrán alcanzar el objetivo planteado.

Derivado de las altas concentraciones poblacionales en las ciudades, se produce un proceso de urbanización acelerado y disperso, que generan externalidades como fallas del mercado y que podrán ser reducidas con el diseño de eficientes políticas públicas, cuyo objetivo debe ser reducir las distancias reales al interior de estos espacios y, por lo tanto, incrementar las economías de aglomeración con las consecuentes mejoras en productividad y bienestar mejorando la accesibilidad urbana, para obtener familias con disponibilidad de empleos, insumos, servicios de educación, salud y la localización de hogares e infraestructura de transporte, que permitan movilizarse a costos razonables, es decir, mejor acceso a trabajos formales, viviendas dignas, educación, desarrollo cultural y salud de calidad, entre otros (CAF, 2017:34).

Las políticas públicas se deben centrar en la solución de distintas alternativas a los problemas y la eficiencia, como parte de la inclusión del tema en la agenda de las instituciones, tomando en consideración que toda política pública, requiere del ejercicio de la responsabilidad social y el de los actores de una sociedad, dado que involucra un conflicto de valores dentro de ella, derivado de una diversa percepción en términos de beneficios y costos para los distintos sectores, lo que hace aún más difícil su implementación, políticas diseñadas en función de problemas con metas, impactos y cambios que sean perceptibles para la ciudadanía, necesitando para eso de indicadores que puedan evaluar eficientemente la gestión pública, en términos de eficacia, eficiencia y efectividad de las políticas públicas adoptadas.

Para América latina, se hace rápido adoptar normas que se encuentren en consenso, la dificultad presente consiste en la aceptación por parte de la sociedad, así como en la aplicación de políticas y acciones que hagan cumplir tal legislación.

El desarrollo de una ciudad depende de gran manera de la forma en que sus habitantes se movilizan, pero al mismo tiempo esta movilidad depende de los recursos (económicos e institucionales) con los que cuenta una sociedad, por lo que se hace primordial la elaboración de políticas de movilidad urbana, a fin de obtener bienestar y por ende una mejor calidad de vida para el ciudadano.

Siendo el objetivo fundamental para las habitantes de una ciudad, políticas que mejoren la accesibilidad y el bienestar social, minimizando las externalidades, garantizando las opciones de movilidad para los distintos grupos de la población, entre ellas el uso del suelo, la vivienda y el trabajo. En definitiva, la movilidad en las ciudades debe ser un objetivo central de cualquier política pública y por ende de la gestión de cualquier institución pública.
Existen políticas de acceso a diversos servicios, pero es necesaria la fiscalización en el desarrollo de la ciudad a fin de hacer que se cumpla y que sea funcional.

Según el Reporte de Economía y Desarrollo (RED 2017),

El 39\% de los latinoamericanos se traslada desde su residencia hacia su lugar de trabajo en transporte público, el $22 \%$ en transporte privado y el $26 \%$ a pie. En Europa el $23 \%$ en transporte público, el 54\% en transporte privado y el 11\% a pie. En Estados Unidos el 90\% de los traslados se realizan en automóvil privado (CAF, 2017:60).

donde se puede resumir, que tanto la circulación a pie, como en el transporte público, son los espacios y la infraestructura, hacia dónde deben estar dirigidos y priorizadas las estrategias dentro de cualquier gestión pública. Así, teniendo en consideración que,

la movilidad se asocia con el espacio físico potencialmente alcanzable mediante el desplazamiento, desde un origen determinado y con los medios y vías de transporte disponibles", mientras que "la accesibilidad se refiere a la cantidad y calidad de oportunidades alcanzables para un individuo, en virtud de su movilidad", siendo más compleja que la movilidad (CAF, 2017:122, 123).

Según el Reporte de Economía y Desarrollo (2017) los propósitos de fondo de las políticas de desarrollo urbano son: la productividad, el bienestar o, en síntesis, la accesibilidad (CAF, 2017:59), accesibilidad urbana y una mejor cohesión social, que deberá mejorar con la relación a

tres dimensiones intimamente integradas: el uso de la regulación del suelo, la movilidad y el transporte, y el acceso a vivienda y servicios básicos...asegurando la integración a través de esquemas de gobernanza que tomen en cuenta la necesidad de coordinación en los ámbitos territorial y sectorial (CAF, 2017:58).

La cohesión social es una dimensión sociológica de las ciudades que puede definirse como el grado de consenso de los miembros de un grupo social o, como la percepción de pertenencia a un proyecto o situación común. Hace referencia al grado de convivencia entre los conjuntos de personas con rentas, culturas, edades o profesiones diferentes que viven en una ciudad. Es un estado en el que existe una visión compartida entre los ciudadanos y el Gobierno, obteniendo un modelo de sociedad basado en la justicia social, la primacía del Estado de Derecho y la solidaridad, todos fundados en valores democráticos (IESE Cities in Motion, 2017:12).

El grado de discapacidad del ser humano depende del entorno en el que se desenvuelva, donde se generan obstáculos que impiden o dificultan la independencia, estos elementos son llamados barreras arquitectónicas, considerados elementos que encontramos en el medio físico y que se puede clasificar, en:

1. Barreras del medio natural: presentes en el medio físico natural.

2. Barreras del medio construido (antropizado): presentes en el espacio construido por el hombre.

- Barreras urbanísticas: aquellas que se encuentran en las vías y espacios de uso público.

- Barreras arquitectónicas: aquellas que se encuentran en el acceso e interior de los edificios públicos o privados. 
- Barreras en el transporte: aquellas que se encuentran en los medios de transporte terrestre, aéreo y marítimo.

- Barreras de comunicación: todo impedimento para la expresión y la recepción de mensajes a través de los medios de comunicación o en el uso de los medios técnicos disponibles.

Considerando que, para lograr el consenso y mejores resultados al respecto, se requiere de una planificación coordinada y ordenada para alcanzar propósitos u objetivos, siendo la planificación estratégica y la eliminación de las barreras aspectos fundamentales para la modernización de cualquier gobierno.

\section{ÁREA DE ESTUDIO}

El caso de estudio corresponde al Casco Central y sus adyacencias de la ciudad de Mérida, perteneciente al Municipio Libertador del Área Metropolitana del Estado Mérida en Venezuela, lugar donde encontramos el poder político administrativo, junto con el comercio, una mezcla de residencial y edificaciones de valor patrimonial.

La ciudad de Mérida, conocida formalmente como Santiago de los Caballeros de Mérida, es la capital del municipio Libertador, Campo Elías y Santos Marquina del estado Mérida y una de las principales localidades de los Andes venezolanos. Se ubica en el centro de la región andina, rodeada de imponentes sierras y el pico Bolívar (con 4.987 msnm), el más alto del país, ciudad que resalta como destino turístico dentro del occidente venezolano (Figuras 3 y 4).

El casco central de Mérida en el Municipio Libertador, es un área relativamente pequeña. Ubicada sobre una meseta, la ciudad es estrecha y alargada, con 28 km de extremo a extremo de longitud, y unos 5 km en su punto más ancho, está marcada por 2 ríos, el río Chama en el costado sur-este, borde natural de la meseta y el río Albarregas, que separa la meseta en dos bandas.

Según el Plan de Ordenación urbanística del área metropolitana Mérida-Ejido-Tabay aprobado en el año de 1999 según resolución 3001, el área es considerada como

Área de Valor Tradicional de Mérida (AVT-1). Corresponde a los núcleos tradicionales de la ciudad de Mérida y sus áreas adyacentes, las cuales conjugan su carácter de área de valor histórico urbano arquitectónico con el centro empleador y sedes de actividades residenciales, comerciales, administrativas, gubernamentales, culturales y religiosas del nivel metropolitano. Por constituir un área representativa del área metropolitana; deberá mantener las características formales y volumétricas, los elementos urbanos y estructuras que constituyan documentos representativos del patrimonio edificado.

Dicha área será objeto de un Plan Especial, con el fin de detectar este valor patrimonial de conjunto (POU. Del área metropolitana Resolución 3001, 1999) Figura 5.

El centro histórico, mantiene la trama española de damero, con 8 avenidas que suben/bajan y 54 calles que las cortan en sentido transversal. Es conveniente distinguir la pendiente como factor limitante para el desarrollo de la movilidad, espacios que pasan desde los 1.100 msnm hasta los 2.000 msnm.

El área de estudio se caracteriza por ser el soporte de los servicios que complementan el uso turístico y estudiantil, que además, identifican la vocación del estado Mérida, donde las edificaciones son consideradas de valor arquitectónico tradicional y utilizadas tanto

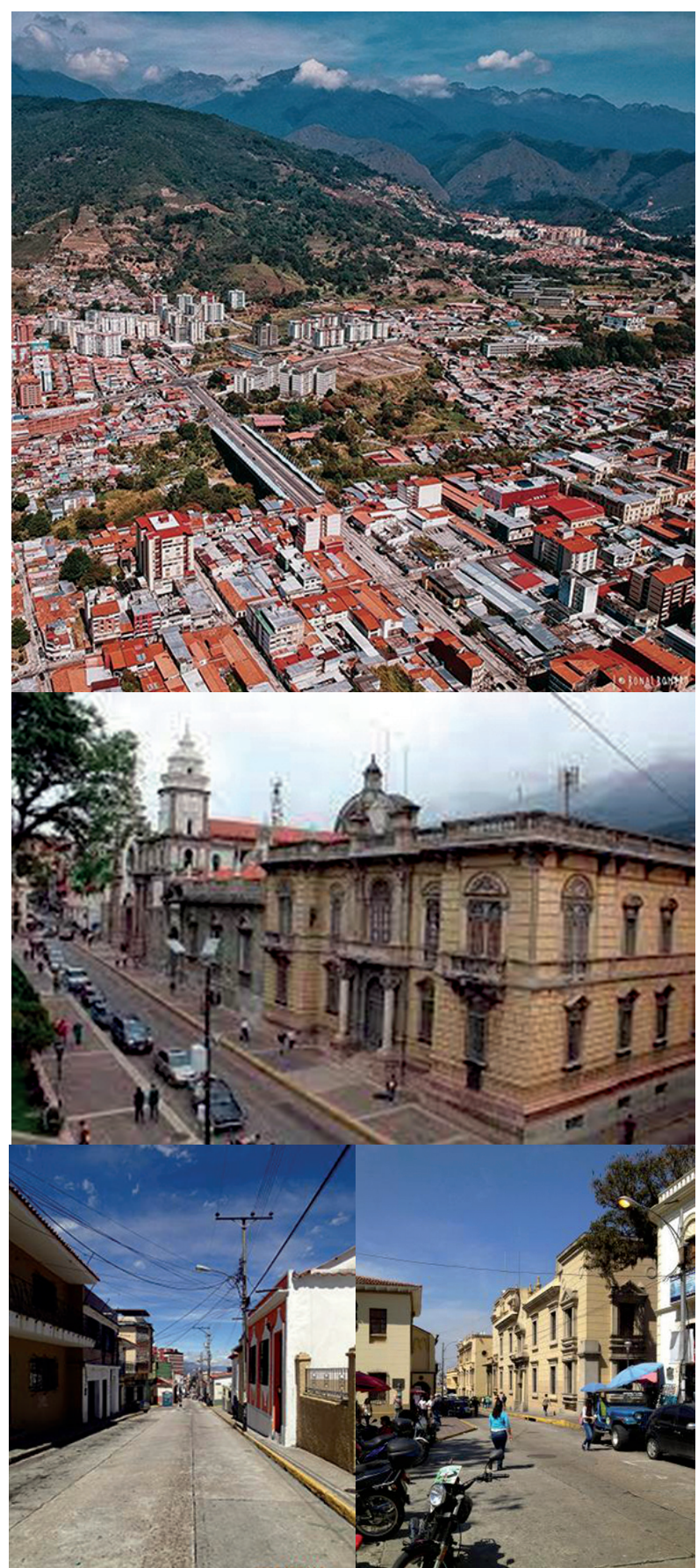

> Figura 3. Imágenes del casco central de la ciudad de Mérida. Nota: en esta imagen se puede observar parte de la estructura urbana, edificaciones resaltantes y bellezas escénicas que le proporcionan la condición turística a la ciudad. Fuente: Elaboración propia. Imagen de Google. 

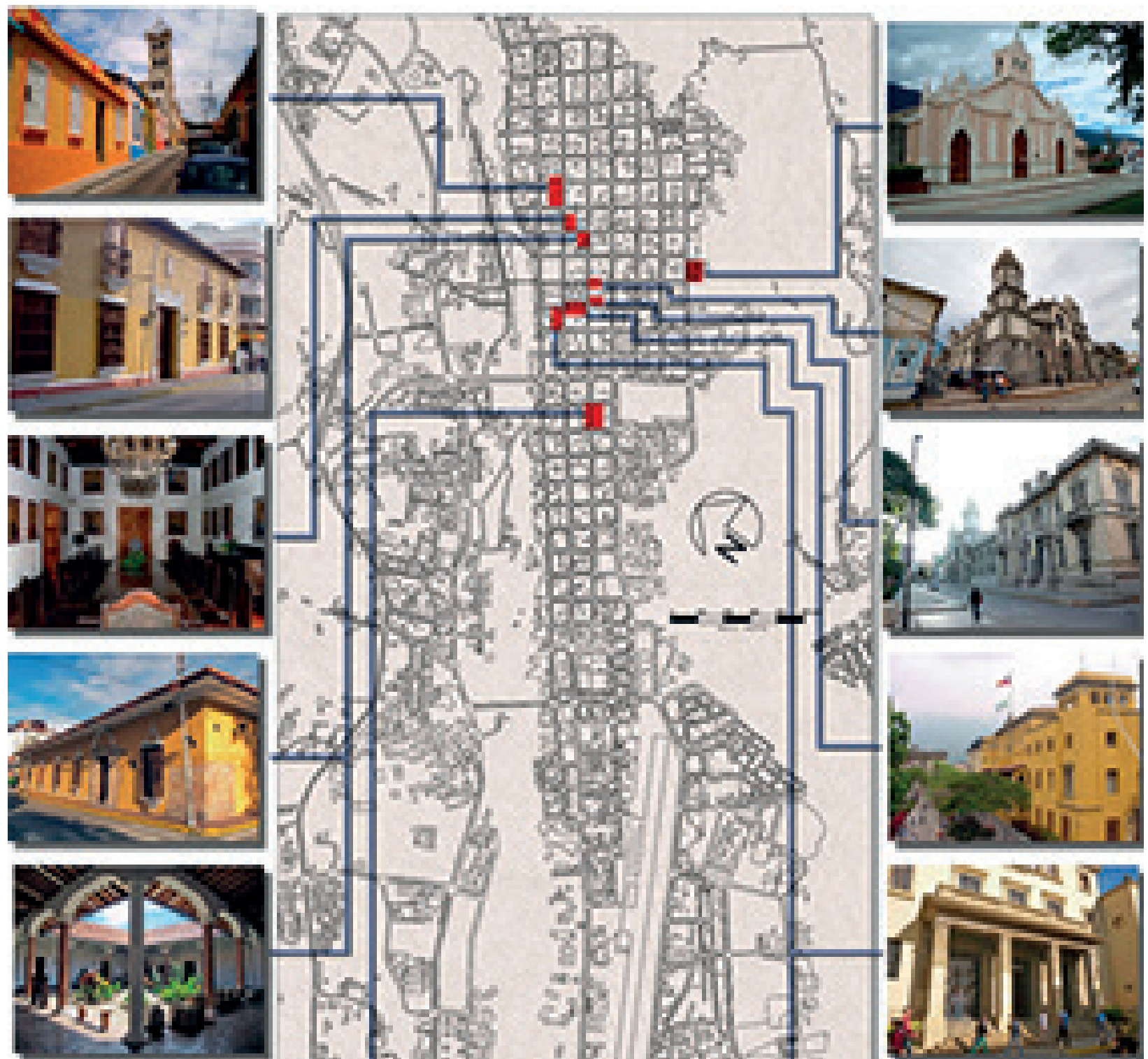

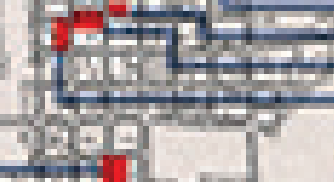
4

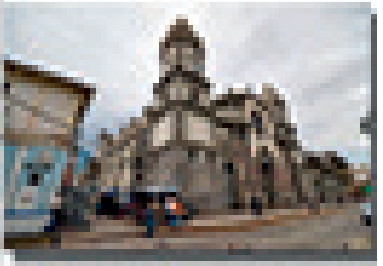

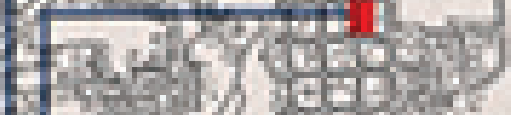

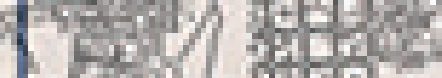
maj Afthis

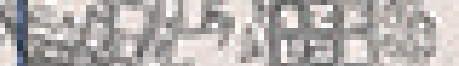
what prest

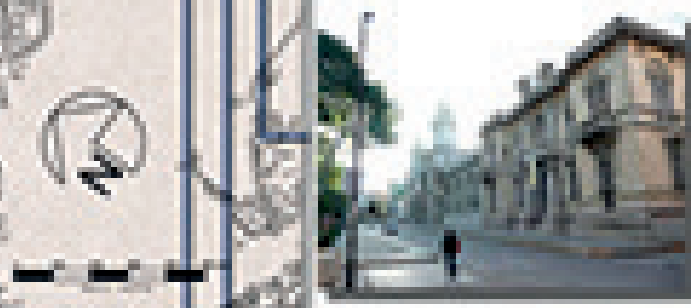

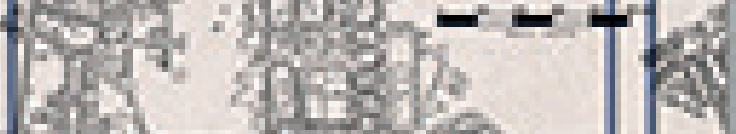
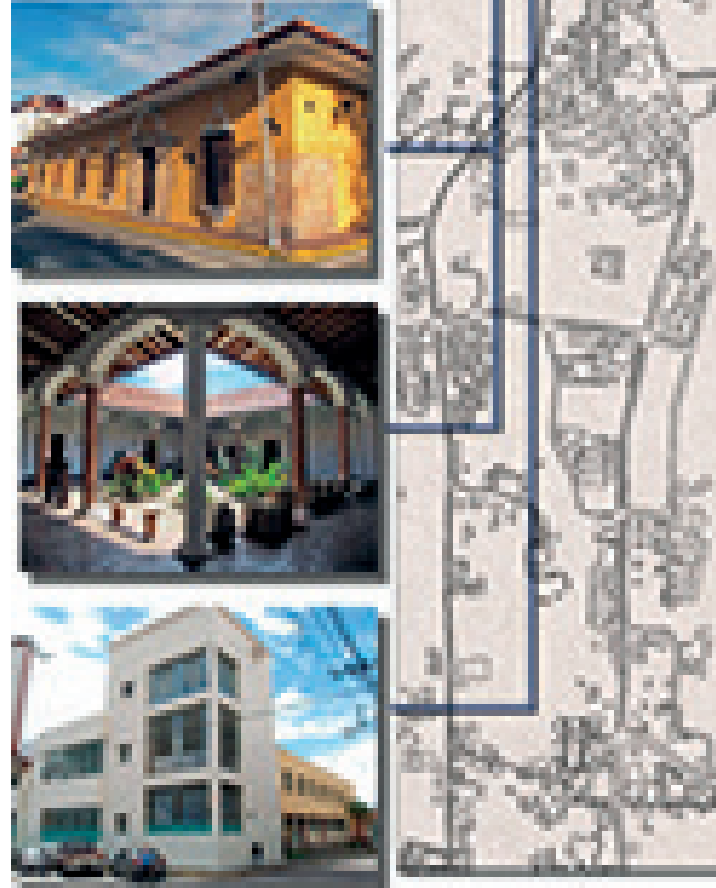

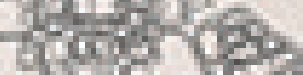
PWD $247 \mathrm{nth}$

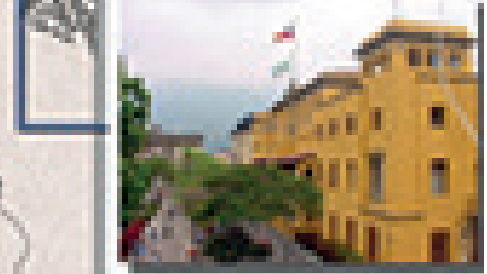
Hix and

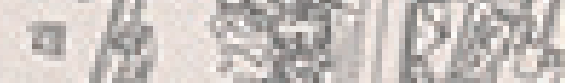
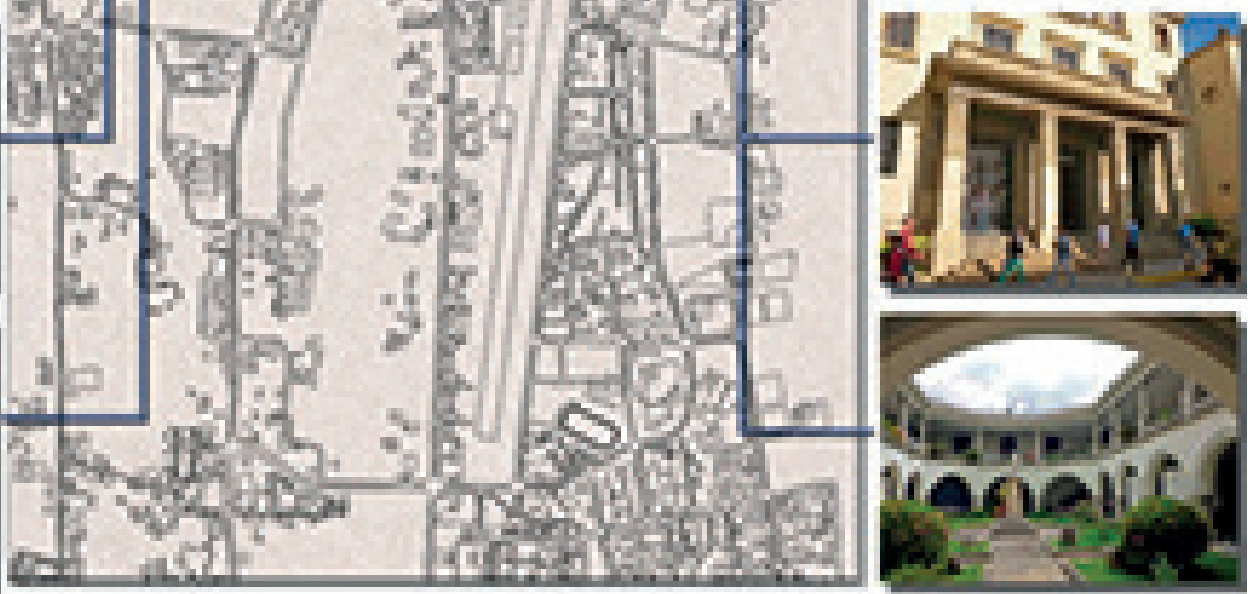

Figura 4. Área de Estudio. Nota: en esta lámina se destaca la estructura urbana de damero que conforma el área central y la ubicación e imágenes de varias edificaciones consideradas como patrimoniales ubicadas dentro del área tradicional. Fuente: Elaboración propia.

$>$ Figura 5. Edificaciones de Valor Tradicional del Casco Central. Nota: en esta imagen se pueden observar algunas de las edificaciones de valor en el área de estudio. Fuente: Imagen de Google. Elaboración propia.

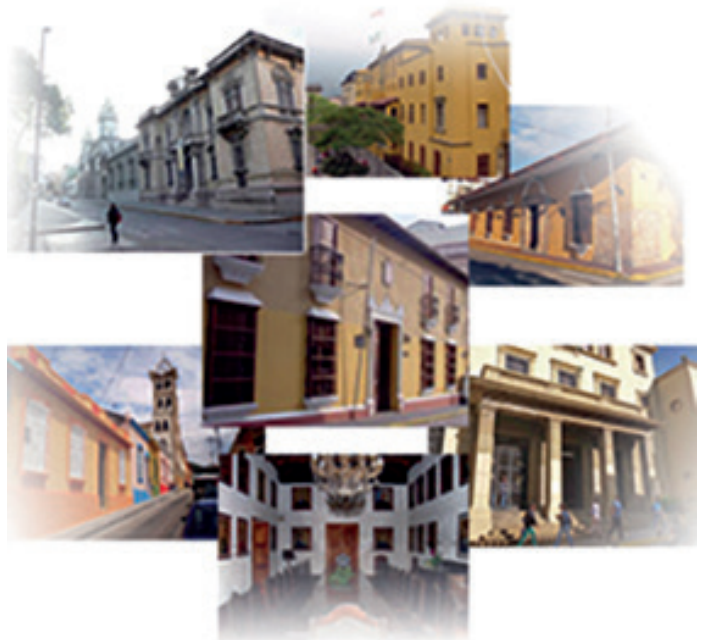


en la actividad administrativa, religiosa, comercial, estudiantil y turística, situación que la ubica como primordial para cualquier tipo de persona, entre las que se puede mencionar, el arzobispado, la gobernación, la catedral, la casa paredes, la casa de los gobernadores, el rectorado de la Universidad de los Andes, Liceo Libertador, iglesia de belén, escuela Rivas Dávila, entre otras casas de estilo colonial que están siendo utilizadas como áreas comerciales.

En lo referente a la legislación venezolana, que involucra accesibilidad, se encuentra la siguiente:

- La Constitución de la República Bolivariana. Proporciona el derecho (de toda persona) acceder a la información y a los datos que sobre sí misma o sobre sus bienes consten en registros oficiales o privados.

- Ley para personas con discapacidad 2007: Quien hace un Ilamado a atender las cualidades y necesidades individuales de las personas con discapacidad. Uso de tecnologías como medios de voz digitalizados y otros sistemas de comunicación.

- Lineamientos sobre el ejercicio pleno del derecho de las personas con discapacidad a una Educación superior de calidad 2007: donde los materiales educativos y software que produzca el Ministerio para la Educación universitaria debe cumplir con las normas de accesibilidad y los principios de diseño universal.

- Ley de Infogobierno. 2013: Desarrollo, implementación y uso de las tecnologías de la información libres, a fin de garantizar a las personas, en igualdad de condiciones, el acceso y la apropiación social del conocimiento asociado a esas tecnologías.

- Gaceta Oficial de la República Bolivariana de Venezuela № 39.633 Portales de Internet accesibles.

- Ley Orgánica de Telecomunicaciones (2000) sus artículos 20 y 50 afirma que se debe garantizar el acceso adecuado de las personas con discapacidad a las redes y servicios en condiciones equiparables a las que se ofrecen al resto de los usuarios.

\section{MÉTODOS}

La metodología utilizada para la investigación consiste en desarrollar mediante un análisis descriptivo del estado del arte, donde se elabora la concreción teórica de los términos de discapacidad, inclusión social, accesibilidad, movilidad como parte del proceso de urbanización en la ciudad y el derecho de sus ciudadanos, en comparación a un simple beneficio otorgado por el Estado, la accesibilidad universal como efecto de la inclusión social, el análisis y comparación de las diferentes estrategias utilizadas por ciudades en tanto en Europa como en América Latina, junto con la evaluación de la accesibilidad en el área de estudio como espacio patrimonial, para finalmente establecer la conclusión de la integración de las etapas anteriores, con la formulación y coordinación de una propuesta de desarrollo de las políticas públicas, desarrollada a través de una planificación estratégica junto al programa de actuaciones, con el fin de mejorar la movilidad y por ende la accesibilidad, mediante la disminución de las barreras arquitectónicas que presenta la ciudad, permitiendo establecer un equilibrio entre el ser humano y su territorio, evaluado la revisión de las condiciones de accesibilidad mediante la identificación de las barreras arquitectónicas, en el área de estudio seleccionada, área central de la ciudad de Mérida, Venezuela.

\section{RESULTADOS}

En función de la clasificación de las barreras definidas anteriormente, fue analizada la situación existente en el área central de
Mérida, evaluando la accesibilidad desde el punto de vista urbanístico, de las edificaciones, el transporte y en las comunicaciones; mediante el recorrido y la observación de las áreas donde se desarrolla la movilidad.

1. Barreras del medio natural: corresponde a las condiciones naturales del sector. Tomando en consideración una longitud promedio, junto a la altura inicial y final, del área de estudio para calcular la pendiente de la meseta encontramos que:

En una longitud de $4823 \mathrm{~m}$, tenemos una altura inicial de 1392 msnm y 1638 msnm con una diferencia de $246 \mathrm{~m}$

encontramos que:

$4823=100 \%$

$246=x$

donde $X=5 \%$ de pendiente del terreno en forma longitudinal.

Por lo que podemos deducir que la pendiente puede ser considerada como limitante para el recorrido longitudinal de una persona con discapacidad.

Evaluando las condiciones transversales, se encontró que para recorrer de un sector denominado la otra banda a la otra parte de la terraza de Mérida, se localiza el río Albarregas con una profundidad entre 10, 20 y 30 m, motivo por el cual se construyeron tres viaductos que conectaron ambos sectores, lo que los identifica como los únicos elementos conectores entre ambos ámbitos.

2. Barreras del medio construido (antropizado): presentes en el medio construido por el hombre:

\subsection{Barreras urbanísticas}

En lo que respecta a lo urbanístico, la ciudad fue desarrollada y creciendo, estableciendo prioridades del vehículo sobre el peatón.

Según la evaluación del recorrido y evaluación del área de estudio, se logró constatar que las aceras, en su gran mayoría, son angostas con anchos entre 0.80 y $0.90 \mathrm{~m}$, y en unas pocas cuadras son inexistentes, o no mantienen la continuidad de sus anchos.

Las plazas y áreas verdes poseen problemas para la accesibilidad, en donde las raíces de los árboles, han levantado el concreto de las áreas de circulación, creando desniveles a lo largo de su tránsito. Al mismo tiempo, dentro del recorrido por la ciudad, se constató la existencia de grandes desniveles en las aceras, con respecto a la senda vehicular, considerando que, junto a la pendiente del terreno, son condiciones que dificultan el libre tránsito de personas con o sin discapacidad.

Adicionalmente, se constató la existencia de obstáculos ubicados en medio de las aceras, tales como los postes de luz, escalones de acceso a edificaciones adyacentes, localización de comercio informal, rampas improvisadas y las raíces de los árboles, que levantan los paños de concreto y limitan el fácil tránsito de cualquier ciudadano, tal como lo podemos observar en las figuras 6 y 7 . (Figuras 6 y 7 ).

\subsection{Barreras arquitectónicas}

En lo que respecta la accesibilidad en las edificaciones, un gran porcentaje de los edificios evaluados se han desarrollado sin tomar en cuenta la topografía, obteniendo de esta manera situaciones en donde la cota de acceso no coincide con la de la calle, originando los desniveles que limitan y que, de igual manera, lo podemos denominar como barreras arquitectónicas, sin la existencia de rampas (Figura 8). 

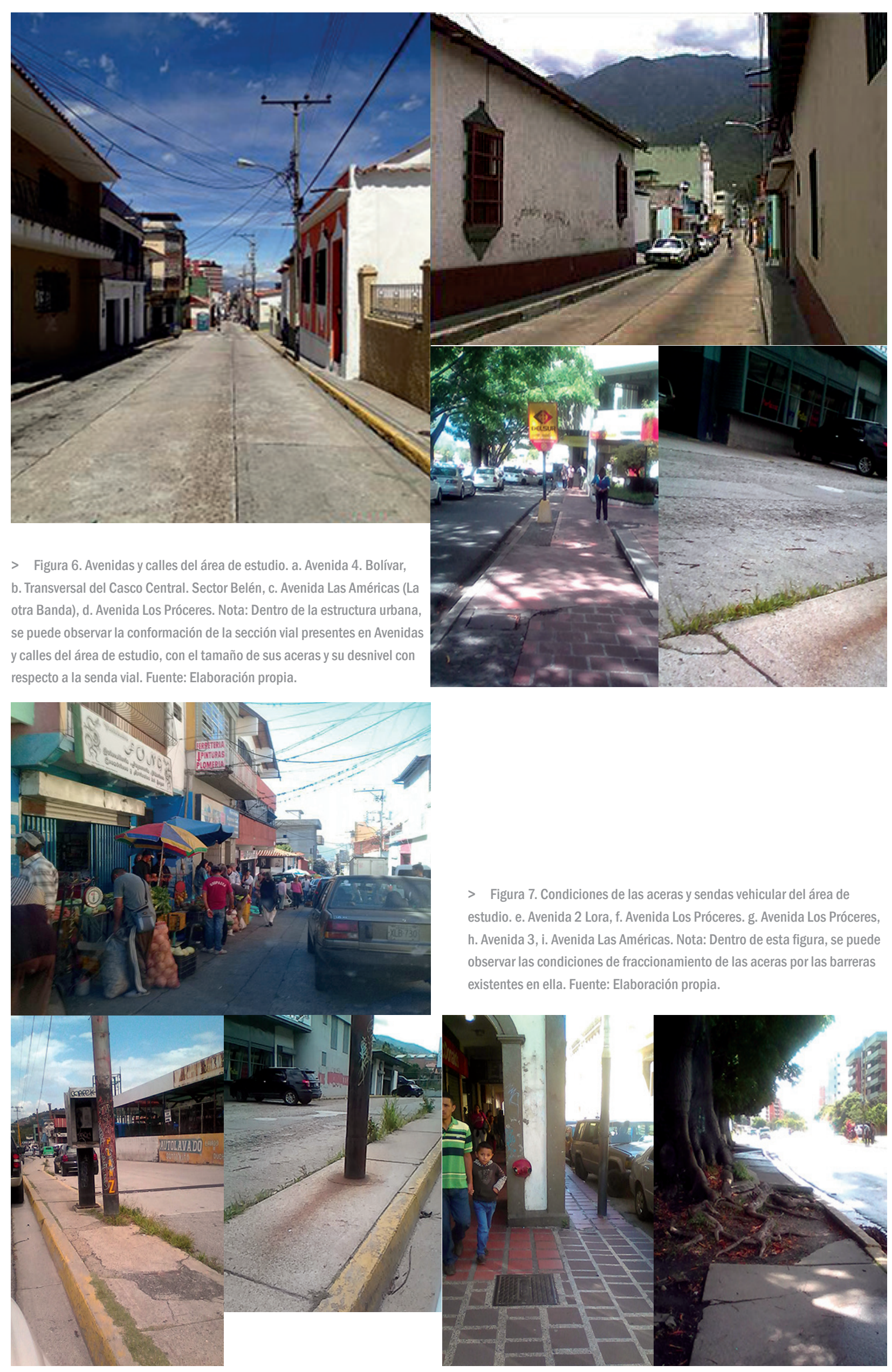

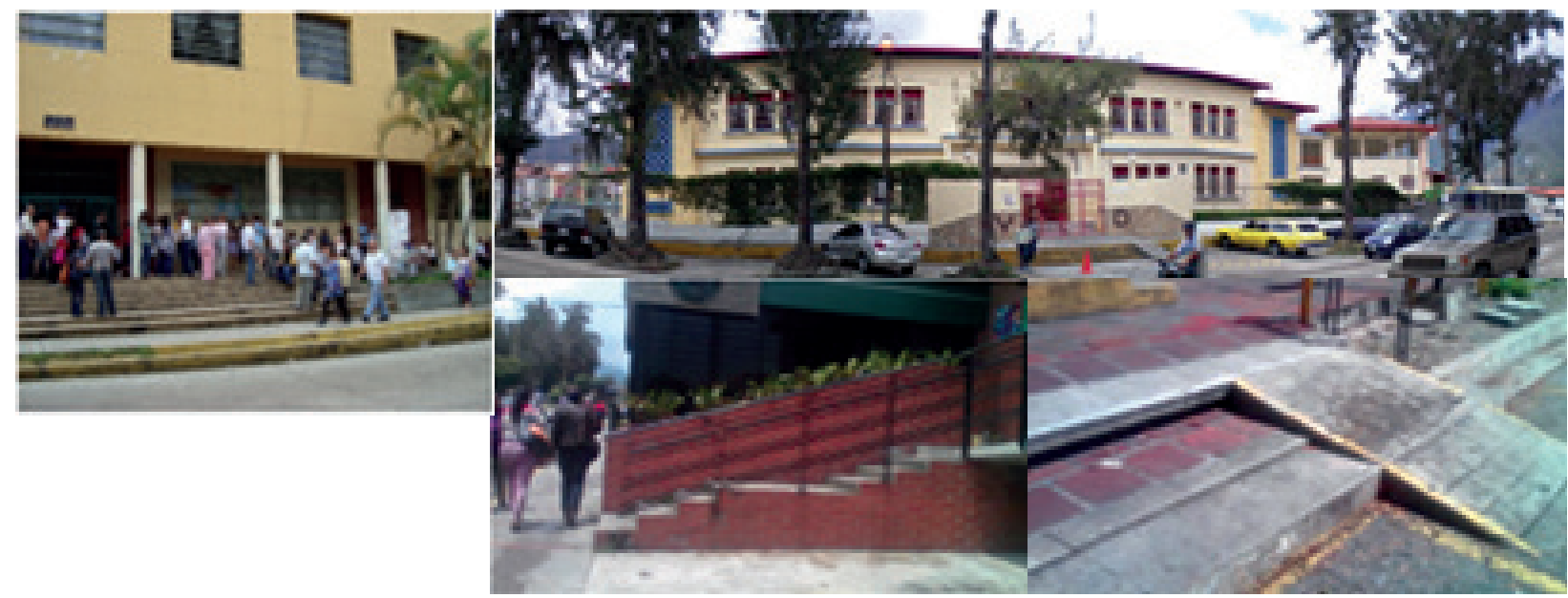

\subsection{Barreras en el transporte}

En cuanto a la accesibilidad del transporte, es de resaltar que ninguna de las líneas de transporte, que funciona en la actualidad posee rampas de accesibilidad para personas con discapacidad, con la excepción del transporte masivo, el cual incorporó estos elementos al diseño del sistema, sin tomar en consideración el contexto, originando una debilidad, para el acceso peatonal, desde cualquier punto de la ciudad (Figura 9).

\subsection{Barreras de comunicación}

En cuanto a la accesibilidad comunicacional, se pudo constatar la falta de elementos comunicacionales, que permitieran el acceso a las personas con determinada discapacidad. Fueron evaluados semáforos, paradas, sistema de transporte público, instituciones, entre otras, encontradas a lo largo de la ciudad.

\section{DISCUSIÓN}

En definitiva, el área de estudio está provista de una serie de legislación que involucra la accesibilidad, sin embargo, se pudo constatar, una fuerte debilidad en la administración local en cuanto a su aplicación.

El acceso de la población a la ciudad, es una igualdad de oportunidades, a una plena participación de las diversas actividades existentes en ellas y que le dan sentido de comunidad, herencia del grupo humano como son los elementos culturales propios de la arquitectura colonial y que se mantiene en el tiempo bajo funciones culturales como los museos, turísticos o comerciales, lo que estimularía aún más la vocación de la ciudad y el sector económico. La disminución de barreras permite a cualquier ciudadano, crecer dentro de una familia, asistir a una escuela, trabajar y participar en esas actividades presentes en ella y en la toma de decisiones de las políticas y estrategias que legisle el municipio.

Para lograr la disminución de las barreras desarrolladas en la ciudad, se hace necesario el desarrollo de una planificación estratégica, concebida bajo los siguientes preceptos:

1. Incluir en el diagnóstico una visión holística del problema con efectos transversales, coordinados por un ente encargado para tal fin, metodología propuesta por la CIF de la Organización Mundial de la salud, que consiste en la caracterización de perfiles de usuarios, con la consideración de condiciones circunstanciales o temporales que podrían implicar dificultades en la movilidad cotidiana en ciertos grupos de usuarios,

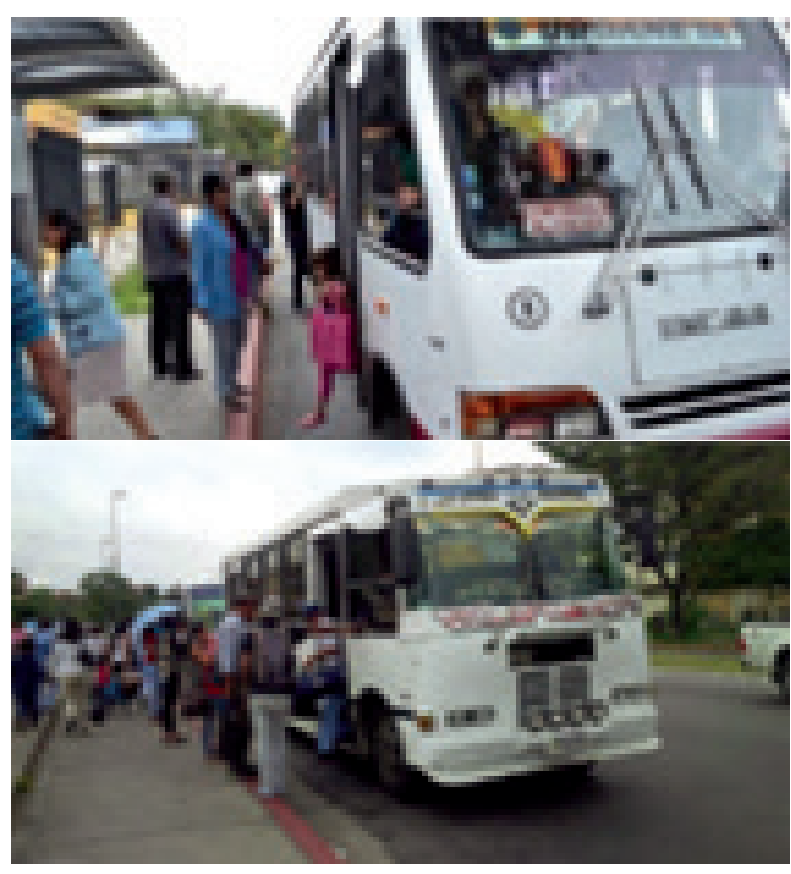

$>$ Figura 8. Escuela Primaria y Secundaria del área de estudio. j. Avenida 4 Liceo Libertador, k. Escuela Básica Vicente Dávila sector Milla, I. Avenida Urdaneta, m. Avenida Las Américas. Nota: En estas figuras constatamos el desnivel entre las aceras, la senda vehicular y la edificación pública educacional, diferencia que oscila entre 1 y $0,5 \mathrm{~m}$ e inexistentes de rampas, barreras que en ocasiones son diseñados con la edificación. Fuente: Elaboración propia.

$>$ Figura 9. Sistema de transporte público del área de estudio. n. Transporte público, Avenida Urdaneta, ñ. Transporte Avenida Las Américas. Nota: En estas imágenes se pudo observar las condiciones poco amigables con la diversidad de personas, tanto de las paradas como del transporte. Fuente: Elaboración propia. 
con perfiles diferentes de discapacidad, junto a la identificación de los tipos de barreras construidas, logrando así una visualización del proceso que sigue una persona para el logro de su objetivo.

2. Establecer una misión con objetivos concretos derivados del diagnóstico de la visualización del proceso.

3. Las políticas resultantes, deberán reflejar distintas visiones, portadoras de valores y responsabilidades dejando claro su finalidad, utilizando una gran variedad de instrumentos que van desde la creación o simulación de mercados, hasta la provisión puramente pública.

4. Definir indicadores que permitan a la población, la evaluación permanente de políticas públicas, para que logren hacer estratégico dicho instrumento.

Las ciudades poseen grandes problemas que, en ocasiones, son el resultado de externalidades negativas cometidas por empresas constructoras y una falta de inspección de la ejecución de sus obras, siendo el municipio el que puede internacionalizar esos problemas, en la búsqueda de soluciones inclusivas y sostenibles.

En definitiva, el área de estudio no se encuentra diseñada ni adaptada para el acceso a personas con determinadas discapacidades, de hecho, en ocasiones se le hace difícil esta accesibilidad para la totalidad de la población, para lo cual debemos hacer reflexiones de la manera que podamos corregir estas limitaciones identificando las barreras arquitectónicas existentes en la ciudad.

\section{CONCLUSIONES}

El centro de negocios de una ciudad, corresponde a un centro de servicios con valor de uso y disfrute para una colectividad, identificados en ocasiones, como patrimonio cultural, que está marcado por la concentración población, manteniendo una identidad y continuidad, que estimula la diversidad y su utilización bajo diferentes funciones administrativas, culturales, comerciales, educacionales, económicas, entre otras, que logran el desarrollo de su vocación, lo que requiere establecer una coordinación de sus políticas de rehabilitación de sus espacios públicos y una regularización de la estructura urbana, las edificaciones y del mobiliario urbano de valor patrimonial, en función de la igualdad de condiciones, a fin de conseguir una accesibilidad universal de sus espacios construidos.

Esta accesibilidad plena, se deriva de la disminución de las barreras del espacio construido, logradas a través de la planificación estratégica, se diagnostique según la clasificación de las barreras construidas, descritas anteriormente y el Mapa de Viaje del cliente (MVC), metodología propuesta por la CIF de la Organización Mundial de la salud (2001), que consiste en la caracterización de perfiles de usuarios; cumpliendo el objetivo de la disminución a la exclusión social, mediante la construcción de espacios físicos, culturales, educacionales, administrativos, sociales y económicos, que empoderen al ciudadano, a una sociedad mucho más igualitaria.

\section{BIBLIOGRAFÍA}

ADAVALOS23 (2008) La efectividad de las políticas públicas a través de la medición de la gestión pública.

Banco Interamericano de Desarrollo (2019) Accesibilidad e inclusión en el transporte. Mapas de viaje Bogotá. Análisis en ciudades latinoamericanas. BID $3^{\text {a }}$ edición.

Bojórquez, Yolanda (2006) Accesibilidad total: una experiencia incluyente desde la arquitectura. Revista electrónica Sinéctica, núm. 29, pp. 43 - 50. Instituto Tecnológico de Estudios Superiores de Occidente, Jalisco, México.

CAF Banco de Desarrollo de América Latina (2017) Reporte de economía y desarrollo RED - 2017. Crecimiento urbano y acceso a oportunidades: un desafío para América Latina, CAF Banco de Desarrollo de América Latina, $3^{a}$ edición, Bogotá, Colombia.

Castro, Carolina; Escobar, Natalia; Leiva, Romina \& Sánchez, Cindy (2015) Estudio de caso: Barreras y facilitadores en el proceso de inserción laboral de personas en situación de discapacidad intelectual que trabajan en el supermercado Unimarc. Universidad UCINF, Facultad de Educación.

Índice IESE Cities in motion (2017) Business School, Índice IESE Cities in motion, 3a edición, Universidad de Navarra.

Linares, Johana; Hernández, Andrea; Rojas, Héctor (2018) Accesibilidad espacial e inclusión social: experiencias en ciudades incluyentes en Europa y Latinoamérica, Civilizar Ciencias Sociales y Humanas, Vol. 18, núm. 35.

Muñoz, Betilde; Barrantes, Alexandra (2016) Equidad e inclusión social: superando desigualdades más inclusivas, Organización de los Estados Americanos, Departamento de inclusión social, Secretaría de acceso a derechos y equidad.

Plan de Ordenación Urbanística del Área Metropolitana Mérida - Ejido - Tabay (1999, febrero 01). Resolución N³001, publicado en Gaceta Oficial Extraordinaria $N^{\circ} 5.303$, Mérida, Venezuela.

Stang, María F. (2011) Las personas con discapacidad en América Latina: del reconocimiento jurídico a la desigualdad real. CEPAL, UNFPA.

Vásquez, Armando (2010) La discapacidad en América Latina, recuperado en https://www.paho.org/uru/dmdocuments/ Discapacidad\%20America\%20Latina.pdf 